\title{
New simulation and reconstruction software for the EUSO pathfinders, with example applications
}

\author{
T.C. Paul ${ }^{* 4}$, M.E. Bertaina ${ }^{1,2}$, F. Bisconti ${ }^{3}$, J. Eser ${ }^{6}$, G. Figueiredo ${ }^{4}$, M. Fouka ${ }^{7}$, \\ F. Guarino ${ }^{8,9}$, L. Del Peral ${ }^{5}$, Z. Sahnoun ${ }^{7}$, for the JEM-EUSO Collaboration \\ E-mail: thomas . paulalehman. cuny • edu \\ ${ }^{1}$ Dipartimento di Fisica, Universita' di Torino, Italy. \\ ${ }^{2}$ Istituto Nazionale di Fisica Nucleare - Sezione di Torino, Italy. \\ ${ }^{3}$ Karlsruhe Institute of Technology (KIT), Germany. \\ ${ }^{4}$ Lehman College, City University of New York, USA. \\ ${ }^{5}$ Universidad de Alcalá (UAH), Madrid, Spain. \\ ${ }^{6}$ Colorado School of Mines, Golden, USA. \\ ${ }^{7}$ Dep. Astronomy, Centre Res. Astronomy, Astrophysics and Geophysics (CRAAG), Algiers, \\ Algeria. \\ ${ }^{8}$ Universita' di Napoli Federico II - Dipartimento di Scienze Fisiche, Italy. \\ ${ }^{9}$ Istituto Nazionale di Fisica Nucleare - Sezione di Napoli, Italy.
}

The Extreme Universe Space Observatory (EUSO) is designed to detect the highest energy particles in the Universe by observing the fluorescence and (reflected) Cherenkov light produced when these ultrahigh energy cosmic rays (UHECR) traverse the Earth's atmosphere. Unlike existing cosmic ray observatories, EUSO will view the atmosphere from above. A number of pathfinder missions have been completed or are in development in order to evaluate the potential of this method, including a prototype instrument flown aboard a stratospheric balloon, a similar instrument co-located with the Telescope Array, an improved instrument to be flown aboard long-duration super pressure balloon, as well as a precursor instrument called Mini-EUSO, which will fly aboard the international space station. Here we describe a relatively new general-purpose software framework, known as Offline, which facilitates detailed simulation and reconstruction of UHECR events and laser and flasher test shots observed by the various EUSO pathfinders. This new software is based on open-source codes developed over roughly a decade by a collaborative effort of several particle astrophysics and high energy physics experiments, and is designed with the needs of large collaborations in mind. We briefly explain the machinery used to manage user contributions to simulation and reconstruction algorithms, organize an abundance of configuration data, and provide access to time-dependent information on detector and atmospheric properties. We show a number of examples of simulation and reconstruction of the EUSO pathfinder data using this software.

35th International Cosmic Ray Conference - ICRC2017

10-20 July, 2017

Bexco, Busan, Korea

* Speaker. 


\section{Introduction}

Large, geographically dispersed scientific collaborations face the challenge of designing a software framework for simulating their instrumentation and performing data analysis such that contributions from many collaborators can be managed under the same framework, and results from different members of the collaboration can be meaningfully compared. The JEM-EUSO Collaboration comprises over 300 scientists from 15 nations, and hence faces these common challenges.

The objective of the EUSO mission is to observe ultrahigh energy cosmic rays (UHECR) from space via measurement of the fluorescence light produced when cosmic ray induced air showers excite atmospheric nitrogen and Cherenkov light reflected from the Earth's surface or from clouds. An overview of the project and the scientific objectives are described elsewhere in these proceedings [1]. The stage is being set for this ambitious project via a number of pathfinder missions, also discussed in these proceedings [2].

From the initial stages of EUSO planning, a software package known as the EUSO Simulation and Analysis Framework (ESAF) [3] was implemented in order to address large-scale simulation and reconstruction challenges. At roughly the same time ESAF development was underway, the Pierre Auger Collaboration [4] was also ramping up its efforts to address the same sorts of challenges. The Auger Collaboration is also large, with over 500 researchers from 16 countries.

Initial implementation of the Auger analysis framework [5], identified by the logo Offlline, began in 2003, and the code has been in continuous use for simulation and reconstruction for the Auger Observatory since 2004. The Offline design has a demonstrated capacity to accommodate many Observatory extensions over a long time scale, and is currently being adapted to the next major upgrade to the Observatory [6]. Indeed, at the time of writing Offline comprises over 380000 lines of code. The Offline framework is freely available upon request, and parts of the code have been adopted by other collaborations, including CODALEMA [7], TUNKA [8], HAWC [9], LOFAR [10] and NA61/SHINE [11].

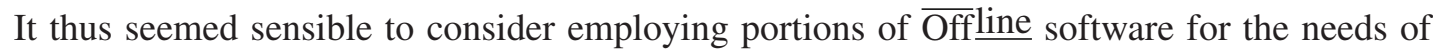
EUSO and the EUSO pathfinders; in particular, the overarching Offline framework, utilities and the well-vetted algorithms related to fluorescence and Cherenkov light emission and propagation are of interest. Taking on board this simulation and analysis package affords the opportunity for code sharing between ESAF and $\overline{\text { Offline }}$ and performing cross-checks. The adaptation of Offline to the needs of EUSO commenced in 2013, and it has since been employed for simulation of the large-scale JEM-EUSO concept [12] as well as simulation and reconstruction of data from the EUSO pathfinders. Here we review the Offline design and present examples of applications to the pathfinder program.

\section{Framework Design}

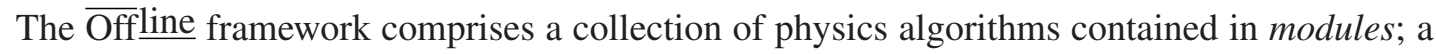
RunController which commands the modules to execute in a particular sequence; a read/write Event Data Model from which modules read information and to which they write their results; a Detector Description which provides an interface to conditions data; and a CentralConfig which directs the 
modules and framework components to their configuration data and which tracks provenance. The general scheme is illustrated in Fig. 1.

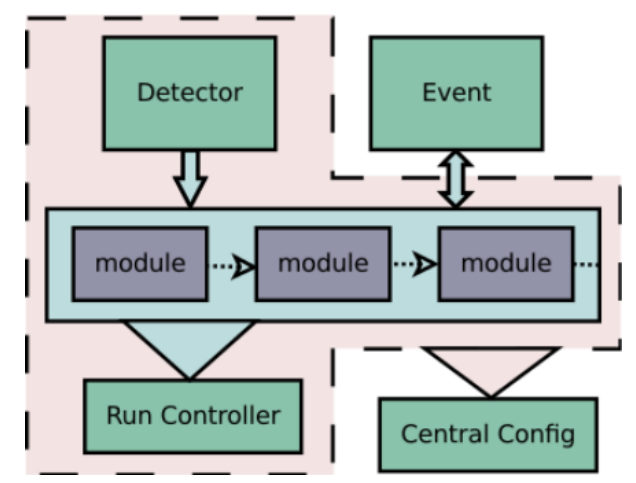

Figure 1: General organization of the Offline framework. See the text for detailed explanation.

Simulation and reconstruction steps are normally organized in a simple pipeline. Collaborators

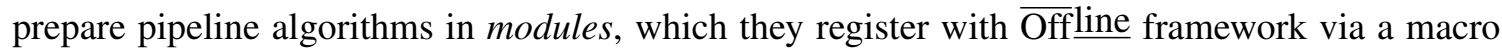
that makes the framework aware of which modules exist for potential use at run time. This modular design facilitates comparison of algorithms prepared by different teams, and supports building a variety of applications by combining modules in various sequences. One can, for instance, swap out a module for reading in simulated showers with a module to simulate laser shots in the instrument field of view (FOV); this can be done at run time with no need for re-compiling any code. Control of module sequences is implemented with a RunController, which directs module execution according to a set of user provided instructions. We devised an XML-based language for specifying sequencing instructions, including support for nested loops and mechanisms for breaking loops in case certain conditions are or are not satisfied; this approach has proved sufficiently flexible for the majority of our applications, and it is simple to use, though more sophisticated Python-based module control is also possible.

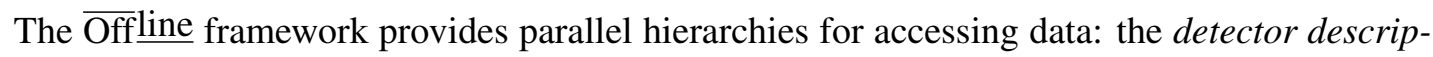
tion for retrieving conditions data, which can include detector materials and geometry as well as time-dependent information such calibration data, background measurements and atmospheric conditions data; a plug-in mechanism in the atmosphere description supporting various ways computing fluorescence and Cherenkov yields as well as Rayleigh and Mie scattering and absorption, using parametric models or measurements stored in databases; and an event data model for reading and writing the simulation and reconstruction data which changes for each event. In the case of the detector description, data requests are relayed to a back-end comprising a registry of so-called managers, each of which is capable of extracting a particular sort of information from a particular data source. This approach helps to keep the unpleasant details of dealing with different formats isolated into code fragments that can be dealt with individually by the relevant experts. The manager mechanism is also configurable at run-time via XML, allowing users to choose which conditions data sources and which conditions analysis algorithms to use for evaluation of the chosen data source. Managers are arranged in a "chain of responsibility" such that if an upstream manager cannot answer a request, it can be passed along to downstream managers for another try. 
The event data model holds the raw, calibrated, reconstructed and Monte Carlo information, and constitutes the backbone for communication between modules. The event is instrumented with a protocol allowing modules to discover its constituents at any point in processing, and thereby determine whether the input data required to carry out the desired processing are available (and

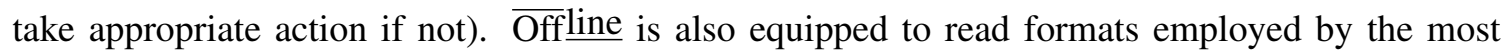
popular air shower simulation packages [13], as well as data formats used by the different EUSO pathfinders.

Offline provides an XML and XML Schema [14] based system to organize and track data used to configure the software for a variety applications as well as to organize parameters used in the physics modules. The Central Config configuration tool points modules and framework components to the location of their configuration data, and connects to Xerces-based [15] XML parsers to assist in reading information from these locations. We have wrapped Xerces with our own interface which provides ease of use at the cost of somewhat reduced flexibility, and which also provides conveniences such as automatic casting to EUSO data containers as well as automatic units conversion, obviating the need to enforce a prescribed units convention.

The Central Config records all configuration data accessed during a run and stores this in an XML log file, which can be subsequently used to reproduce a run with an identical configuration. We have found such provenance tracking to be indispensable for simulation production and data analysis; it nearly always happens that someone eventually needs to look up precisely what configuration was used to produce a simulation or process data.

Syntax and content checking of the configuration files is implemented using W3C XML Schema validation. Taking advantage of industry standards such as XML Schema reduces the amount of code users and developers must prepare to ensure sensible input, and supports very robust checking.

\section{Continuous integration testing, build, and dependency resolution}

It is common enough for bugs to be introduced into software without anyone noticing until much later, making the process of tracking them down extremely tedious. To alleviate this misery, we have implemented both low-level and high-level tests. Low-level tests exercise individual components of the framework, whereas high-level tests run full physics applications. In the case of high-level tests, results from a particular simulation or analysis chain are recorded and compared to the results found the last time the test was run, with an alert sent to developers in case of a change. We automate the testing process using the Jenkins [16] continuous integration system. This tool is

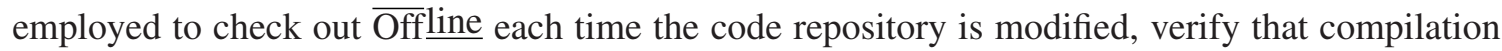
and installation work under a variety of conditions and compilers, run low- and high-level tests, and automatically generate an email alert identifying the guilty parties in case of a failure.

The Offline build system uses the CMake cross-platform build tool [17]. In order to ease in-

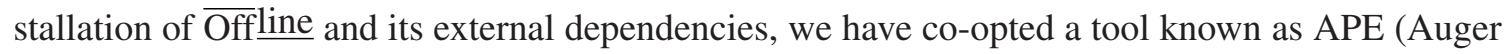
Package and Environment) [18]. APE is a dependency resolution engine co-developed by the Auger and HAWC Collaborations. It automatically downloads a vetted combination of external packages

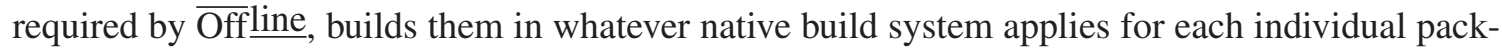
age, and sets up the user's environment. 


\section{EUSO Pathfinders in Offline}

EUSO-balloon: On 25 August 2014, a EUSO prototype comprising a single Photo Detector Module (PDM) and two Fresnel lenses was carried on an overnight stratospheric balloon flight, with launch from Timmins, Canada. The instrument was underflown by a helicopter equipped with a Xenon flasher and a laser which shot a beam across the instrument FOV, allowing for instrumentation testing and development of reconstruction algorithms. The flight is described in more detail elsewhere in these proceedings [19]. Over 250 flasher/laser events were found in the data. Figure 2 displays an Offline reconstruction of an event containing laser light, as well as reconstructed parameters for a subset of the event sample.
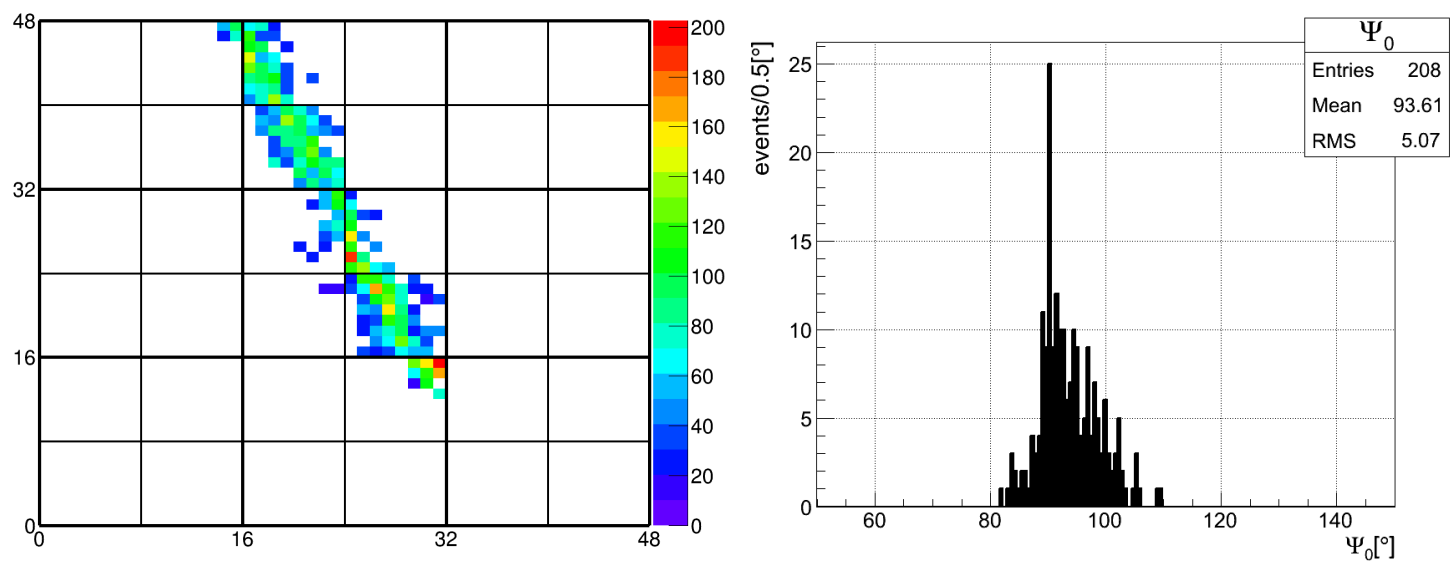

Figure 2: Left. EUSO-Balloon focal surface image of a laser shot. The horizontal and vertical axes label the pixel number, and the color indicates the number of counts integrated over all of the time gates $(2.5 \mu \mathrm{s}$ each) for which flasher or laser data appeared to be present. Right. Reconstructed laser tracks for shots fired horizontally across the EUSO-Balloon FOV. Note this distribution should peak at $90^{\circ}$ for horizontal tracks.

EUSO-TA: An instrument similar to that carried by EUSO-Balloon is co-located with the Telescope Array (TA) Black Rock Mesa (BRM) site. This prototype, called EUSO-TA, can record laser shots from fixed and roving laser facilities at TA as well as UHECR air showers using the BRM telescopes to trigger EUSO-TA. Simulation and reconstruction of EUSO-TA events were performed in a joint effort using both the ESAF and Offline packages, as detailed elsewhere in these proceedings [20].

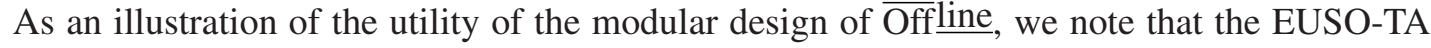
background has been simulated using two methods (so far), one using Poissionian fluctuations to mimic background, and one using in situ measurements of the instrument performance. Figure 3 shows the focal surface with simulated background plus a simulated laser event as well as the laser track after reconstruction algorithms are applied.

EUSO-SPB: On 25 April 2017 an improved prototype instrument comprising 1 PDM and 2 lenses was launched on a NASA Super Pressure Balloon (SPB) from Wanaka, New Zealand, accumulating 10 days of exposure. One of the goals of EUSO-SPB was to make the first observations of UHECR from above. Like the EUSO-balloon pathfinder described previously, this instrument has been simulated in $\overline{\mathrm{Off}} \underline{\text { line }}$, and development of reconstruction algorithms is underway. As discussed 

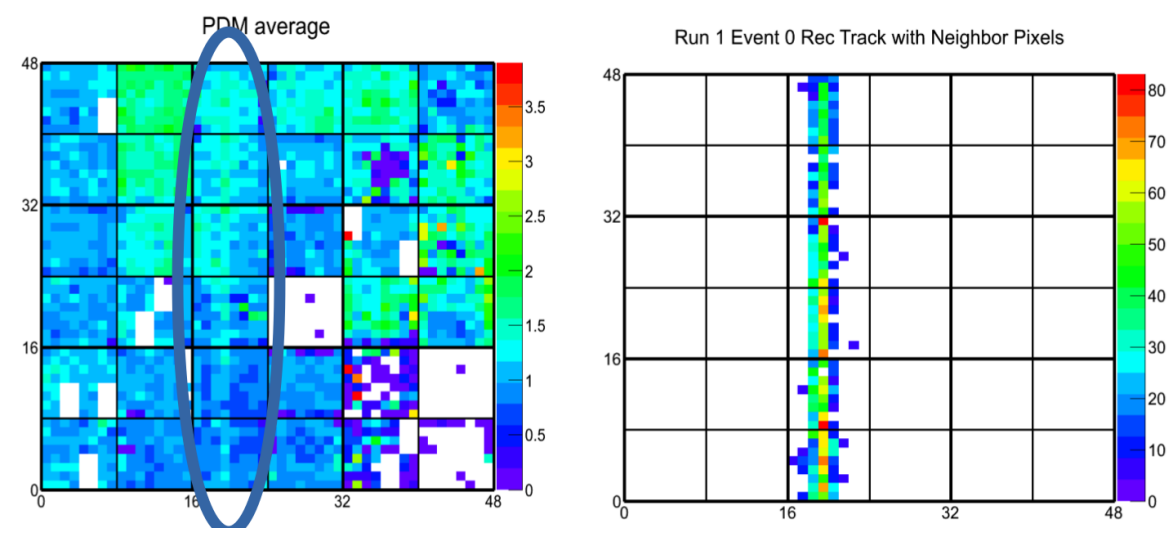

Figure 3: Left: Simulated laser shot ( $9 \mathrm{~mJ}$ at $34 \mathrm{~km}$ distance) together with background simulation integrated over 128 time gates. The laser signal is inside the blue oval. Right: Laser shot extracted from background

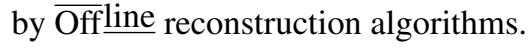

in $\S 2$, Offline was designed with the machinery to straightforwardly access time-dependent conditions data. This will be important for EUSO-SPB reconstruction, as time-dependent background and calibration information are required for the data analysis. Further details on the EUSO-SPB mission appear in these proceedings [21].

Mini-EUSO: The Mini-EUSO instrument comprises a compact 2 lens telescope with a $\pm 19^{\circ}$ field of view. It will be deployed on board the International Space Station (ISS), and view the Earth's atmosphere through a UV transparent window from within ISS. In addition to serving as a EUSO proof-of-principle, Mini-EUSO will also be used to study airglow, atmospheric phenomena like Transient Luminous Events, meteors and meteoroids and perhaps record a few UHECR events. A Geant4-based [24] simulation of the instrument has been prepared in Offline, with raytracing at several incident angles depicted in Figure 4. Further details on Mini-EUSO are available in these proceedings [22].

Proposed EUSO-SPB2: Another potential SPB mission employing EUSO-like instrumentation has been disussed in the literature [23]. The detector described therein, called EUSO-SPB2, is designed not only to detect fluorescence light produced by UCECR, but also Cherenkov light generated by nearly horizontal air showers. The Offline and ESAF software packages have been used in conjunction to estimate an expected UHECR event detection rate of around 5 events for a flight with about 138 dark hours, though the final numbers will depend on details of the instrument design and performance.

\section{Summary}

Best practices for design of simulation and analysis codes have evolved during the advent of large, geographically dispersed collaborations. Here we have briefly outlined adaptation of Offline software originally designed for the Pierre Auger Collaboration to suit the requirements of the ongoing EUSO pathfinder missions, as well projecting performance of possible future missions.

Acknowledgment: This work was partially supported by US NSF grant PHY-1205854, NASA grant NNX13AH52G, 


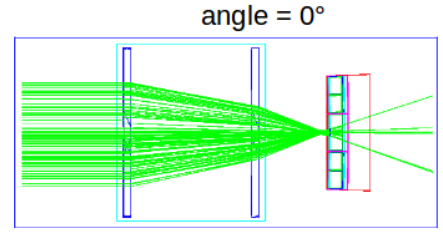

angle $=5^{\circ}$

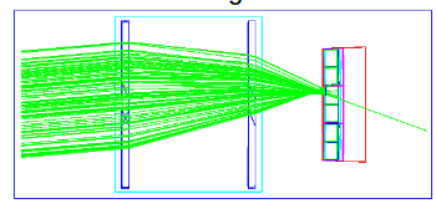

angle $=10^{\circ}$

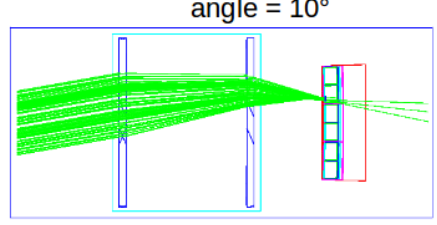

angle $=15^{\circ}$

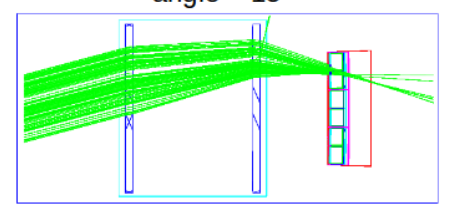

angle $=20^{\circ}$

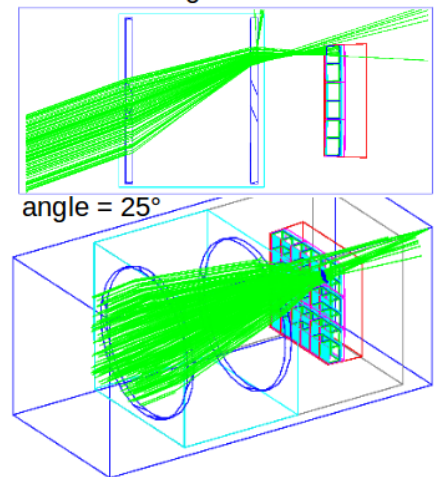

Figure 4: Geant4-based raytracing for the mini-EUSO optical system for point sources at various angles with respect to the optical axis.

by the Italian Ministry of Foreign Affairs, General Direction for the Cultural Promotion and Cooperation, by the 'Helmholtz Alliance for Astroparticle Physics HAP' funded by the Initiative and Networking Fund of the Helmholtz Association, Germany. The Spanish Consortium involved in the JEM-EUSO Space Mission is funded by MICINN \& MINECO under the Space Program projects: AA2009-06037-E/AYA, AYA-ESP2010-19082, AYA-ESP2011-29489C03, AYA-ESP2012-39115-C03, AYA-ESP2013-47816-C4, MINECO/FEDER-UNAH13-4E-2741, CSD2009-00064 (Consolider MULTIDARK) and by Comunidad de Madrid (CAM) under projects S2009/ESP-1496 \& S2013/ICE-2822.

\section{References}

[1] M. Casolino, "The EUSO mission to study UHECR from space: status and perspectives from the flights of EUSO-SPB and MINI-EUSO on the International Space station," these proceedings (ICRC 2017 ID:).

[2] K. Shinozaki et al., "Implications for space-based UHECR observation from UV background studies by JEM-EUSO pathfinders," these proceedings.

[3] C. Berat et al., "ESAF: Full Simulation of Space-Based Extensive Air Shower Detectors," Astropart.Phys. 33 (2010) 221.

[4] A. Aab et al. [Pierre Auger Collaboration], "The Pierre Auger Cosmic Ray Observatory," Nucl. Instrum. Meth. A 798, 172 (2015) doi:10.1016/j.nima.2015.06.058 [arXiv:1502.01323 [astro-ph.IM]].

[5] S. Argiro, S. L. C. Barroso, J. Gonzalez, L. Nellen, T. C. Paul, T. A. Porter, L. Prado, Jr., M. Roth et al., 'The Offline Software Framework of the Pierre Auger Observatory," Nucl. Instrum. Meth. A 580, 1485 (2007) [arXiv:0707.1652 [astro-ph]];

P. Abreu et al. [AUGER Collaboration], "Advanced functionality for radio analysis in the Offline software framework of the Pierre Auger Observatory," Nucl. Instrum. Meth. A 635, 92 (2011) [arXiv:1101.4473 [astro-ph.IM]].

[6] A. Aab et al. [Pierre Auger Collaboration], "The Pierre Auger Observatory Upgrade - Preliminary Design Report," arXiv:1604.03637 [astro-ph.IM]. 
[7] D. Ardouin, A. Belletoile, D. Charrier, R. Dallier, L. Denis, P. Eschstruth, T. Gousset, F. Haddad et al., "CODALEMA: A cosmic ray air shower radio detection experiment," Int. J. Mod. Phys. A21S1, 192-196 (2006).

[8] B. A. Antokhonov, D. Besson, S. F. Beregnev, N. M. Budnev, O. B. Chvalaev, A. Chiavassa, O. A. Gress, N. N. Kalmykov et al., "A new 1-km**2 EAS Cherenkov array in the Tunka valley," Nucl. Instrum. Meth. A639, 42-45 (2011).

[9] A. U. Abeysekara et al. [HAWC Collaboration], "The HAWC Gamma-Ray Observatory: Design, Calibration, and Operation,” arXiv:1310.0074 [astro-ph.IM].

[10] http://www.lofar.org/

[11] N. Angrily et al. [NA61 Collaboration], "NA61/SHINE facility at the CERN SPS: beams and detector system,” JINST 9, P06005 (2014) [arXiv:1401.4699 [physics.ins-det]].

O. Wyszynski, A. Laszlo, A. Marcinek, T. Paul, R. Sipos, M. Szuba, M. Unger and D. Veberic, "Legacy code: Lessons from NA61/SHINE offline software upgrade adventure," J. Phys. Conf. Ser. 396, 052076 (2012).

R. Sipos, A. Laszlo, A. Marcinek, T. Paul, M. Szuba, M. Unger, D. Veberic and O. Wyszynski, “The offline software framework of the NA61/SHINE experiment," J. Phys. Conf. Ser. 396, 022045 (2012).

[12] J. H. Adams et al. [JEM-EUSO Collaboration], "An evaluation of the exposure in nadir observation of the JEM-EUSO mission,” Astropart. Phys. 44, 76 (2013) doi:10.1016/j.astropartphys.2013.01.008 [arXiv:1305.2478 [astro-ph.HE]].

[13] R. Engel, D. Heck and T. Pierog, "Extensive air showers and hadronic interactions at high energy," Ann. Rev. Nucl. Part. Sci. 61, 467 (2011);

S. J. Sciutto, “AIRES: A System for air shower simulations. User's guide and reference manual. Version 2.2.0," astro-ph/9911331;

T. Bergmann, R. Engel, D. Heck, N. N. Kalmykov, S. Ostapchenko, T. Pierog, T. Thouw and K. Werner, "One-dimensional Hybrid Approach to Extensive Air Shower Simulation," Astropart. Phys. 26, 420 (2007) [astro-ph/0606564];

H. J. Drescher and G. R. Farrar, "Air shower simulations in a hybrid approach using cascade equations,” Phys. Rev. D 67, 116001 (2003) [astro-ph/0212018].

[14] http://www.w3.org/standards/xml/schema

[15] http://xerces.apache.org/

[16] https://jenkins.io/

[17] http://www. cmake.org

[18] https://svn.auger.unam.mx/trac/projects/ape/

[19] M. Bertania et al., "Results of the EUSO-Balloon flight," these proceedings.

[20] F. Bisconti et al., "Simulation study of the detected and expected events for the EUSO-TA fluorescence detector" these proceedings.

[21] L. Wiencke et al., "EUSO-SPB Mission and Science," these proceedings.

[22] M. Ricci, "Mini-EUSO: a precursor mission to observe and study Atmosphere and Earth UV emission from the International Space Station," these proceedings.

[23] J. H. Adams et al., "White paper on EUSO-SPB2," arXiv:1703.04513 [astro-ph.HE].

[24] https://geant4.cern.ch 